\title{
Serial Sectioning in SEM: Challenges and Opportunities
}

Amir Avishai ${ }^{*}$, Nanthawan Avishai ${ }^{*}$, Grahame Kidd ${ }^{* *}$, Keith Olszens $^{* * *}$, David McDonald ${ }^{* * *}$, Sillas Duarte $^{* * * *}$, Debarshi Mustafi ${ }^{* * * * *}$, Krzysztof Palczewski ${ }^{* * * * *}$, Sharon Frase ${ }^{* * * * * *}$, and Arthur H. Heuer*

*Dept. of Mat. Sci. and Eng., Case Western Reserve University, Cleveland, OH, 44106.

**Dept. of Neurosciences, Lerner Research Institute, Cleveland Clinic Foundation, Cleveland, Ohio 44120.

***Dept. of Molecular Bio. \& Microbiology, CWRU School of Medicine, Cleveland, OH, 44106.

****Dept. of Restorative Sciences, Herman Ostrow School of Dentistry, University of Southern

California, Los Angeles, CA, 90089.

*****Dept. of Pharmacology, Case Western Reserve University, Cleveland, OH, 44106.

******Cell and Tissue Imaging Center, St. Jude Children's Research Hospital, Memphis, TN 38105.

Over the last few years, in situ serial sectioning combined with scanning electron microscopy (SEM) has been gaining increasing interest [1-8]. This imaging modality fills a gap between TEM tomography and 3D light microscopy [1,5-7]. With image resolutions in the nanometer range and fields of view that may extend over a few hundred microns, these methods have made probing into the sub-cellular range and its inner structure readily possible, and allowed detailed 3D investigation from the organelle level to the cellular level to become easily accessible.

Intricate 3D structures such as; brain tissue [1,7,8], viral particles in dendritic white blood cells [3], or photoreceptor cell architecture are just a few examples of targets of these methods, allowing researchers to trace and follow how interwoven features fit together in a larger context. The resolution and volume analyzed with these methods, together with increased automation of image acquisition increasing i.e. ease of data collection, promise to make these methods indispensable.

Two approaches currently dominate this niche: serial ion ablation (SIA)-SEM [1-4], and serial block face (SBF)-SEM [5-8]. The former involves sectioning of the tissue using a Ga ion beam, while the latter makes use of an in situ ultra-microtome to remove sections. Although the two methods can be interchanged in some cases, each has it merits and therefore are complementary.

The focused ion beam (FIB) approach or SIA-SEM has a several advantages: it is highly site specific [4], it allows the user to section through materials with very different properties (hard and soft tissue for example), and work on non-ideal/rough surfaces can be carried out [4]. Figure 1 shows a dendritic white blood cell (DC) having captured HIV particles (green) in the membrane folds of the DC. The biggest drawback however, is the limitation in the field of view that may be exposed by FIB sectioning and the milling time it requires. A common practice to reduce milling time when larger sections are necessary is to increase the ion beam current used. However, this can result in coarser cuts and beam damage to sensitive materials such as a plasticembedded tissue. SBF-SEM on the other hand, allows for a very large field of view, up to a 
square millimeter, and the cutting time is not size dependent. The increased section size, together with relatively poor conducting properties in many cases, may lead to charging during SEM imaging. For this reason such ultra-microtome systems are typically mounted on variable pressure microscopes that allow charge compensation.

Both methods are destructive in nature and therefore before one runs an experiment, careful attention must be given to consider the experimental parameters of the setup. Typical consideration should include image resolution, field of view, slice thickness, and overall volume to be analyzed.

This presentation will attempt to clarify potential strengths of each method, current limitations, and possible future trends.

\section{References}

[1] G. Knott, et. al., The Journal of Neuroscience, 28(12), (2008) 2959.

[2] G. Kidd et. al., Microscopy Today, 18(01), (2010) 18.

[3] N. Avishai et. al., Microsc. Microanal., 16 (Suppl 2), (2010) 1098.

[4] J.A. W. Heymann, et. al., Journal of Structural Biology, 155, (2006) 63.

[5] K.L. Briggmann and W. Denk, Current Opinion in Neurobiology, 16, (2006) 562.

[6] W. Denk, and H. Horstmann, PLoS Biology, 2(11), (2004) e329.

[7] Moritz Helmstaedter et. al., Current Opinion in Neurobiology, 18, (2008) 633.

[8] G.A. Perkins et al., The Journal of Neuroscience, 20, 30(3), (2010) 1015.

[9] The aid of Ben lich, Rick Passey, Brandon Van Leer, Scott Shawmeker (FEI), and Joel Mancuso (Gatan) is gratefully acknowledged.

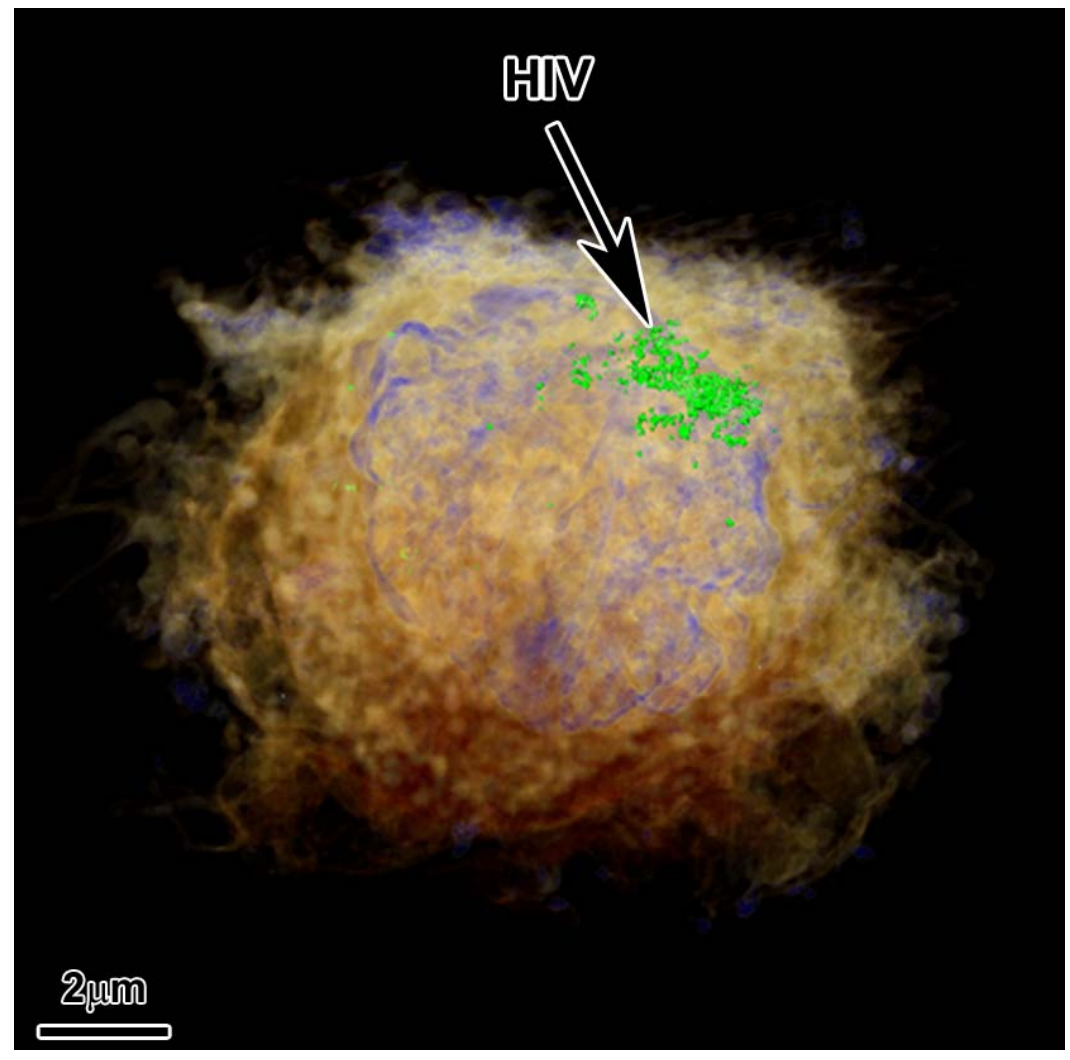

Figure 1.3D modeling of DC with HIV particles stored within surfaceconnected membrane Folds. 